\title{
The relevance of monitoring of antibodies against the polycyclic aromatic hydrocarbon (PAH) and PAH-DNA adducts in serum in relation to lung cancer and chronic obstructive pulmonary disease (COPD)
}

\author{
N. PAUK ${ }^{1, \star}$, S. KLIMESOVA ${ }^{1}$, J. KARA ${ }^{1}$, J. TOPINKA ${ }^{2}$, J. LABAJ ${ }^{3}$ \\ ${ }^{1}$ Department of Pneumology and Thoracic Surgery, Hospital Na Bulovce, Prague, 3rd Faculty of Medicine Charles University Prague, Czech \\ Republic; ${ }^{2}$ Laboratory of Genetic Ecotoxicology, Institute of Experimental Medicine, AS CR, Prague, Czech Republic; ${ }^{3}$ Research and Development \\ Department, Vidia Ltd., Jesenice, Czech Republic
}

*Correspondence: pauknorb@yahoo.com

Received June 25, 2012/ Accepted September 24, 2012

\begin{abstract}
Certain substances from the polycyclic aromatic hydrocarbons (PAHs) group are major inducers of respiratory tract carcinogenesis. The presented are the results of a serological epidemiological study aimed at monitoring the levels of anti-PAH antibodies and antibodies to PAH-DNA adducts in serum. The patients studied belonged both to the group of those with known lung disease (COPD and lung cancer), as well as to the healthy population of people who due to the work conditions or those at the place of residence can expect increased exposure to PAHs. In addition to the results proper that confirm increase of the genotoxic exposure risk to PAH in smoke-polluted places of residence and other PAH polluted environments. There has also been proved the relevance of still commonly used markers (DNA adducts), as well as the suitability of new markers, more favourable from the economic and practical viewpoints (anti-benzo(a)pyrene-7,8-dihydrodiol-9,10-epoxide-DNA [anti-BPDE-DNA], anti-Benzo(a)pyrene antibodies of the IgA class).
\end{abstract}

Key words: polycyclic aromatic hydrocarbons (PAHs), anti-PAH antibodies, DNA adducts, antibodies against PAH-DNA adducts, COPD, lung cancer

The polycyclic aromatic hydrocarbons (PAH or PAHs) belong to the group of aromatic hydrocarbons with at least two benzene cores, which arise mainly during the incomplete combustion.

A major source of $\mathrm{PAH}$ pollution are industrial enterprises (chemical plants, foundries, power plants, heating plants), but also the internal combustion engines of vehicles or local heating [1]. In the indoor environment an important source of PAHs can be smoking, burning of candles or incense, or heat treatment of food by grilling or frying $[1,2]$.

Some substances in this group are among the most significant inducers of carcinogenesis, not only in the respiratory tract (lungs, trachea and larynx), but also in other organs such as kidneys, urinary bladder, oesophagus and pancreas [3].

PAHs are not genotoxic per se, but must be metabolized by enzymes from a family of cytochrome P450 (1A1, 1A2) to electrophilic compounds capable of reacting with nucleophilic centres of DNA molecules. It is a multistage process of activation as well simultaneous detoxification of these substances.
Enzymes involved in metabolic transformations are genetically controlled, and therefore the entire rate of activation and detoxification of the chemical substances depend on the genotype of the cell. Following the activation, the PAHs link to the DNA nucleotides, resulting in the production of DNA adducts that can give rise to mutations during the DNA replication process $[2,6]$. Besides production of the DNA adducts the PAHs are capable to cause oxidative damage to the DNA, chromosomal aberrations and participate in the molecular changes in oncogenes and tumour suppressor genes [7]. Man is exposed to PAHs during life process, and in people with greater exposure, a production of antibodies against PAHs has been shown [8].

If increased PAH exposure causes formation of DNA adducts in the cells, it also forms antibodies to PAH-DNA adducts [8]. Method for detection and quantification of these antibody levels in human blood (serum), in clinical practice could serve as a biomarker of PAH exposure and could also help in predicting PAHs induced cancer risk. On the prin- 
ciple of immunochemical reaction PAH induced antibodies may balance out any other present PAHs and thus indirectly contribute to reducing the amount of PAHs entering the cells where they could subsequently be activated to genotoxic metabolites. If however these metabolites, which are more hydrophilic than unchanged PAHs, have already been produced, they are then easily excreted from the cell into the extracellular space, where they are balanced out by specific antibodies thus preventing their further activation of a significantly more reactive form [9]. The long-term chronic exposure to PAHs (for instance, by smoking) reduces body immune response and presumably reduces levels of anti-PAH antibodies $[4,5]$. It therefore leaves organism increasingly vulnerable to negative effects of PAHs from the environment.

The serum-epidemiological studies which would investigate carcinogen-specific antibodies relation to the risk of developing cancer in the exposed population are not yet developed [9]. The objective of this study is to provide a baseline for further retrospective and prospective environmental epidemiological studies addressing PAH exposition. It investigates the presence of anti-PAH antibodies and antibodies to PAH-DNA adducts in serum in relation to smoking, place of residence and prevalence of lung diseases and cancer.

\section{Patients and methods (Evaluation of epidemiology study)}

Study population. The data and samples for this study were collected in years $2006-2010$. The study was focused on levels of anti-PAH antibodies and antibodies to PAH-DNA adducts in serum of both patients with known lung disease and healthy individuals, in whom higher exposition to $\mathrm{PAH}$ was expected due to place of residence or occupation.

In patient group, the smokers represent the group of people who smoked at least 100000 cigarettes in total. In group of healthy individuals the smokers were defined as exsmokers without regard to amount of smoked cigarretes.

The patients were recruited from Department of Pneumology and Thoracic Surgery, Na Bulovce Hospital. The first part consisted of patients with a new diagnosis of lung cancer (regardless of its micromorphological characteristic) prior to anticancer therapy. The other part of the patients enrolled had a diagnosis of chronic obstructive pulmonary disease (COPD), a serious non-cancerous lung disease which occurs mainly due to smoking and other long-term respiratory exposure to chemical substances.

In constructing the group of healthy people with increased exposure to PAHs, individuals who are exposed to emissions from transport due to their professional occupation (bus drivers and workers of garages of the public transport in Prague) were incorporated to presented study. As well as the inhabitants of the Ostrava-Karvina region, an area with the greatest long-term concentrations of PAHs, especially benzo(a)pyrene $(\mathrm{B}(\mathrm{a}) \mathrm{P})$ in air were also included. By measuring the concentrations of PAHs in the Czech Republic in 2005, the carcinogenic benzo(a)anthracene showed its annual averages ranging from
0.8 to $10.2 \mathrm{ng} \cdot \mathrm{m}^{-3}$. Specialized stations had measured high concentrations of $\mathrm{B}(\mathrm{a}) \mathrm{P}$ in Ostrava and Karvina. In winter, on certain days it was observed that the average daily concentration constituted of more than $30 \mathrm{ng} . \mathrm{m}^{-3}$ [1]. These patients were non-smokers or former smokers with insignificant smoking history. Based on the detection of cotinine in the blood (a marker showing the recent presence of nicotine in the body), we have labelled them as non-smokers. Implementation of sampling and data collection was conducted in collaboration with the Laboratory of Genetic Ecotoxicology, Institue of Experimental Medicine (IEM) Academy of Sciences CR.

Data collection and statistical methods. All study participants signed their informed consent and filled in questionnaires for biostatistical data processing. The questionnaires were focused on issues of smoking, inhalation exposure to PAHs from the surrounding environment (at work and home, and the possibility of influencing the levels of PAHs by consumption of food and dietary supplements). Overall, the study used 210 blood samples of healthy subjects and 190 patient samples. All samples were used for detection of antibodies against benzo(a)pyrene of IgG and IgA class, antibodies to BPDE-DNA adducts in serum and determination of the total DNA adducts in lymphocytes isolated from the blood of the subjects of the study. Individual antibodies were determined using ELISA kits designed by the Research and Development Department of the Vidia Company Ltd. and determination of DNA adducts was carried out using the ${ }^{32} \mathrm{P}$-postlabelling method in the Laboratory of Genetic Ecotoxicology, IEM Academy of Sciences CR.

During the statistical processing there was constructed a linear model for each measured variable (IgG, IgA, anti-adducts and adducts) using predictors that were available for subjects in all groups (according to the year of birth, group, gender, municipality size, heating, proximity to roads or other sources of air pollution, occupation, type of workplace, pollutants in the workplace, both active and passive smoking - stratified by the length of period, type and frequency of smoking tobacco products, the consumption of meat, milk, cheese, fried foods, smoked foods, vegetables, fruits, vitamins, tea and coffee). Of all the predictors, an appropriate one for each model was selected.

There was used a stepwise algorithm (gradual addition of features to the set already used for prediction) with the AIC criterion (Akaike information criterion - regression quality criterion based on the value of the residual sum of squares - RSS, penalized by the number of variable $n$ ). Standard statistical analysis due to asymmetric distribution of the data was not possible to use, therefore the appropriate data transformation was necessary to perform. There was used the Box-Cox transformation. The parameter of the transformation was determined by Maximum Likelihood Metod. The transformation is applicable only for positive data. The positive constant was added to the zero value of anti-DNA adducts.

For comparison of the anti-adducts to adducts, Spearman's rank correlation coefficient (serial correlation coefficient) 
was calculated. The calculation represents a non-parametric statistical characteristic. In this case the correlation between the two variables is characterized by pairs of values arranged according to the size. It is especially suitable in situations in which the variable distribution does not meet the Gaussian distribution. The confidence interval for the propabilily of value occurrence is 95\%. The value of the Spearman's rank correlation coefficient is minimally dependent on devianted values.

The measurement. In these types of biological studies the DNA adducts in peripheral lymphocytes are commonly used as determination markers of genotoxic DNA damage, using the ${ }^{32} \mathrm{P}$-postlabelling method. However, as the studies performed on mice show, no increased levels of DNA adducts may be recorded due to rapid repair processes [10]. In addition, this method is burdened with many complications. It is time consuming and its use is possible only in laboratories with adequate safety level due to the presence of radioactive isotope of phosphor.

Immunoglobulin IgG is known as a representative of the humoral immune response against the toxins that can be neutralized by creating immunocomplex while activating the complement via the classical pathway. All this would be to the obvious advantage in the case of PAHs inhalation exposure. However based on the results obtained in this study, which monitored the levels of individual classes of anti-PAH antibodies in serum of the observed population, we conclude that in this case the organism selects protection provided by the mucosal antibodies of the IgA class, which are produced by $B$ lymphocytes normally contained in the mucous membranes of the respiratory and gastrointestinal tract. The levels of antibodies against B(a)P of the IgA class correlated well with the levels of DNA adducts as markers of genotoxic damage to DNA, at the group level.

It was revealed by the biostatistical evaluation of the results that another possible determination parameter of genotoxic exposure to PAHs could be the determination of anti-BPDEDNA adducts in serum. These antibodies appear in the body as the immune response to the presence of BPDE-DNA adducts as an antigen. The level of these antibodies increased with repeated exposure to $\mathrm{B}(\mathrm{a}) \mathrm{P}$ and was detectable even when BPDE-DNA adducts determined by the ${ }^{32} \mathrm{P}$-postlabelling method disappeared.

ELISA kits for detection of antibodies against $\mathrm{B}(\mathrm{a}) \mathrm{P}$ of the IgA class and BPDE-DNA adducts in human and mice serum would potentially serve to identify the presence of DNA adducts. It would also reflect the current state of the organism after exposure to PAHs. The determination of these antibodies due to the simplicity, speed and lower financial cost of the process could be applied especially when screening large exposed populations, where altogether difficult determination of DNA adducts by the ${ }^{32} \mathrm{P}$-postlabelling method is impracticable. In case of antibody levels deviant from the "normal“, the results can be applied in the prevention of early reversible changes in the organism.

\section{Results and discussion}

After the biostatistical processing of results stated in the tables 1 to 7 (all values are related to the positive serum value and expressed -in arbitrary units A.U.), in the group of healthy subjects there was found a major increase in the level of DNA adducts, as well as in antibodies against DNA adducts and mucosal anti-B(a)P antibodies of the IgA class in comparison with diagnosed patients. The levels of antibodies against $\mathrm{B}(\mathrm{a}) \mathrm{P}$ of the IgG class had not been changing (see Table 1). In presented study the comparison of the healthy exposed population with healthy unexposed population was not performed.

The importance of environmental influences is also reflected in the increased levels of both DNA adducts and antibodies against DNA adducts. The DNA adducts are detected in peripheral lymphocytes in people who spend considerable amount of time in smoke-polluted environments. The increased levels of both parameters represent the possible genotoxic risk associated with passive smoking (see Table 2 and 3).

Table 1. Comparison of the group of the healthy population (0) versus the group of patients, regardless of the diagnosis (12)

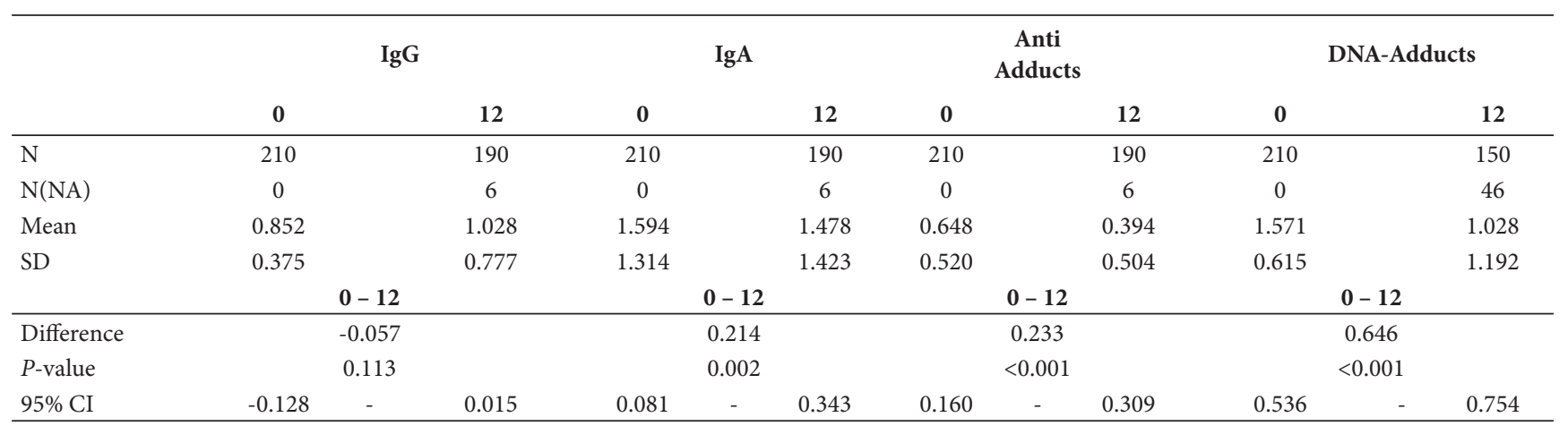

The table 1 shows the significant difference of the level of anti-PAH IgA antibodies (IgA), antibodies against DNA adducts (Anti Adducts) and DNA-Adducts between the group of healthy population (0) and the whole group of patients (1+2). In the case of PAH antibodies of $\operatorname{IgG}$ class (IgG), there were not observed a statistically significant difference between the groups and the values did not show any correlation with other tested parameters. 
Regarding the vitamin supplementation, the data showed that the consumption of vitamins has influence on the level of anti-B(a)P IgA antibodies. In respondents with higher vitamins consumption, the lower levels of anti-B(a)P IgA antibodies were detected (see Table 7). It is in accordance with generally accepted protective functions of vitamins with antioxidant properties.
Patients received mostly multivitamin preparations in tablets (the information is in the medical record), in healthy individuals were not different types of vitamins and their formulation unfortunately were not distinguished in the questionnaires.

No difference was demonstrated in the tested parameters (IgG, IgA, anti-DNA adducts and DNA adducts) when

Table 2. Comparison of the group of the healthy population (0) versus the group of patients with COPD (1)

\begin{tabular}{|c|c|c|c|c|c|c|c|c|}
\hline & \multicolumn{2}{|c|}{ IgG } & \multicolumn{2}{|c|}{$\operatorname{IgA}$} & \multicolumn{2}{|c|}{$\begin{array}{c}\text { Anti } \\
\text { Adducts }\end{array}$} & \multicolumn{2}{|c|}{ DNA-Adducts } \\
\hline & $\mathbf{0}$ & 1 & $\mathbf{0}$ & 1 & $\mathbf{0}$ & 1 & $\mathbf{0}$ & 1 \\
\hline $\mathrm{N}$ & 210 & 65 & 210 & 65 & 210 & 65 & 210 & 53 \\
\hline $\mathrm{N}(\mathrm{NA})$ & 0 & 2 & 0 & 2 & 0 & 2 & 0 & 14 \\
\hline Mean & 0.852 & 1.127 & 1.594 & 1.557 & 0.648 & 0.377 & 1.571 & 1.329 \\
\hline \multirow[t]{2}{*}{ SD } & 0.375 & 0.886 & 1.314 & 1.596 & 0.520 & 0.356 & 0.615 & 1.848 \\
\hline & \multicolumn{2}{|c|}{$0-1$} & \multicolumn{2}{|c|}{$0-1$} & \multicolumn{2}{|c|}{$0-1$} & \multicolumn{2}{|c|}{$0-1$} \\
\hline Difference & \multicolumn{2}{|c|}{-0.107} & \multicolumn{2}{|c|}{0.255} & \multicolumn{2}{|c|}{0.215} & \multicolumn{2}{|c|}{0.560} \\
\hline$P$-value & \multicolumn{2}{|c|}{0.058} & \multicolumn{2}{|c|}{0.013} & \multicolumn{2}{|c|}{$<0.001$} & \multicolumn{2}{|c|}{$<0.001$} \\
\hline $95 \%$ CI & -0.226 & 0.003 & 0.059 & 0.443 & 0.108 & 0.320 & 0.372 & 0.736 \\
\hline
\end{tabular}

Table 3. Comparison of the group of the healthy population (0) versus the group of patients with lung cancer (2)

\begin{tabular}{|c|c|c|c|c|c|c|c|c|}
\hline & \multicolumn{2}{|c|}{ IgG } & \multicolumn{2}{|c|}{$\operatorname{Ig} A$} & \multicolumn{2}{|c|}{$\begin{array}{c}\text { Anti } \\
\text { Adducts }\end{array}$} & \multicolumn{2}{|c|}{ DNA-Adducts } \\
\hline & $\mathbf{0}$ & 2 & 0 & 2 & $\mathbf{0}$ & 2 & 0 & 2 \\
\hline $\mathrm{N}$ & 210 & 125 & 210 & 125 & 210 & 125 & 210 & 97 \\
\hline $\mathrm{N}(\mathrm{NA})$ & 0 & 4 & 0 & 4 & 0 & 4 & 0 & 32 \\
\hline Mean & 0.852 & 0.977 & 1.594 & 1.437 & 0.648 & 0.402 & 1.571 & 0.863 \\
\hline \multirow[t]{2}{*}{ SD } & 0.375 & 0.712 & 1.314 & 1.329 & 0.520 & 0.567 & 0.615 & 0.527 \\
\hline & \multicolumn{2}{|c|}{$0-2$} & \multicolumn{2}{|c|}{$0-2$} & \multicolumn{2}{|c|}{$0-2$} & \multicolumn{2}{|c|}{$0-2$} \\
\hline Difference & \multicolumn{2}{|c|}{-0.035} & \multicolumn{2}{|c|}{0.192} & \multicolumn{2}{|c|}{0.246} & \multicolumn{2}{|c|}{0.683} \\
\hline$P$-value & \multicolumn{2}{|c|}{0.371} & \multicolumn{2}{|c|}{0.012} & \multicolumn{2}{|c|}{$<0.001$} & \multicolumn{2}{|c|}{$<0.001$} \\
\hline $95 \% \mathrm{CI}$ & -0.112 & 0.044 & 0.045 & 0.336 & 0.161 & 0.333 & 0.565 & 0.800 \\
\hline
\end{tabular}

Effects of long term exposure to PAHs from the environment is presented in tab. 2 and 3. There is shown the statistically significant difference in the level of anti-PAH IgA antibodies (IgA), antibodies against DNA adducts (Anti Adducts) and DNA adducts between the the group of healthy population (0) andthe group of patients with COPD diagnosis (1) (tab. 2) and between the group of healthy population (0) and the patients with lung cancer diagnosis (2) (tab.3).

Table 4. Comparison of the group of patients with lung cancer (2) versus the group of patients without lung cancer (1) (patients with COPD)

\begin{tabular}{|c|c|c|c|c|c|c|c|c|c|c|}
\hline & \multicolumn{3}{|c|}{ IgG } & \multicolumn{3}{|c|}{ IgA } & \multicolumn{2}{|c|}{$\begin{array}{c}\text { Anti } \\
\text { Adducts }\end{array}$} & \multicolumn{2}{|c|}{ DNA-Adducts } \\
\hline & 1 & & 2 & 1 & & 2 & 1 & 2 & 1 & 2 \\
\hline $\mathrm{N}$ & 65 & & 125 & 65 & & 125 & 65 & 125 & 53 & 97 \\
\hline $\mathrm{N}(\mathrm{NA})$ & 2 & & 4 & 2 & & 4 & 2 & 4 & 14 & 32 \\
\hline Mean & 1.127 & & 0.977 & 1.557 & & 1.437 & 0.377 & 0.402 & 1.329 & 0.863 \\
\hline \multirow[t]{2}{*}{ SD } & 0.886 & & 0.712 & 1.596 & & 1.329 & 0.356 & 0.567 & 1.848 & 0.527 \\
\hline & \multicolumn{3}{|c|}{$1-2$} & \multicolumn{3}{|c|}{$1-2$} & \multicolumn{2}{|c|}{$1-2$} & \multicolumn{2}{|c|}{$1-2$} \\
\hline Difference & \multicolumn{3}{|c|}{0.069} & \multicolumn{3}{|c|}{-0.063} & \multicolumn{2}{|c|}{0.037} & \multicolumn{2}{|c|}{0.122} \\
\hline$P$-value & \multicolumn{3}{|c|}{0.308} & \multicolumn{3}{|c|}{0.533} & \multicolumn{2}{|c|}{0.324} & \multicolumn{2}{|c|}{0.231} \\
\hline $95 \% \mathrm{CI}$ & -0.059 & - & 0.216 & -0.267 & - & 0.147 & -0.034 & 0.121 & -0.066 & 0.332 \\
\hline
\end{tabular}

Regarding to the comparison of patients with lung cancer (2) and patients with COPD (1), the statistically significant difference in followed parameters was not observed. 
Table 5. Comparison of the group of smokers with COPD (K1) and smokers patients with lung cancer (K2).

\begin{tabular}{|c|c|c|c|c|c|c|c|c|}
\hline & \multicolumn{2}{|c|}{ IgG } & \multicolumn{2}{|c|}{ IgA } & \multicolumn{2}{|c|}{$\begin{array}{c}\text { Anti } \\
\text { Adducts }\end{array}$} & \multicolumn{2}{|c|}{ DNA-Adducts } \\
\hline & K1 & $\mathrm{K} 2$ & K1 & $\mathrm{K} 2$ & K1 & $\mathrm{K} 2$ & K1 & K2 \\
\hline $\mathrm{N}$ & 55 & 106 & 55 & 106 & 55 & 106 & 43 & 84 \\
\hline N(NA) & 1 & 3 & 1 & 3 & 1 & 3 & 13 & 25 \\
\hline Mean & 1.195 & 0.960 & 1.589 & 1.363 & 0.398 & 0.407 & 1.473 & 0.852 \\
\hline \multirow[t]{2}{*}{$\mathrm{SD}$} & 0.940 & 0.716 & 1.618 & 1.167 & 0.373 & 0.589 & 2.009 & 0.536 \\
\hline & \multicolumn{2}{|c|}{$\mathrm{K} 1$ - K2 } & \multicolumn{2}{|c|}{$\mathrm{K} 1-\mathrm{K} 2$} & \multicolumn{2}{|c|}{$\mathrm{K} 1-\mathrm{K} 2$} & \multicolumn{2}{|c|}{$\mathrm{K} 1-\mathrm{K} 2$} \\
\hline Difference & \multicolumn{2}{|c|}{0.144} & \multicolumn{2}{|c|}{-0.009} & \multicolumn{2}{|c|}{0.053} & \multicolumn{2}{|c|}{0.226} \\
\hline$P$-value & \multicolumn{2}{|c|}{0.078} & \multicolumn{2}{|c|}{0.935} & \multicolumn{2}{|c|}{0.244} & \multicolumn{2}{|c|}{0.044} \\
\hline $95 \% \mathrm{CI}$ & -0.012 & 0.295 & -0.235 & 0.214 & -0.032 & 0.144 & 0.003 & 0.457 \\
\hline
\end{tabular}

The level of DNA adduct of the patients with diseases related to smoking (COPD patients - smokers) (K1) are signifantly higher in comparison with the smoker patients with lung cancer (K2).

Table 6. Comparison of the group of non-smoker- (NK1) and smoker- (K2) patients with COPD.

\begin{tabular}{|c|c|c|c|c|c|c|c|c|}
\hline & \multicolumn{2}{|c|}{ IgG } & \multicolumn{2}{|c|}{$\operatorname{IgA}$} & \multicolumn{2}{|c|}{$\begin{array}{c}\text { Anti } \\
\text { Adducts }\end{array}$} & \multicolumn{2}{|c|}{ DNA-Adducts } \\
\hline & K1 & NK1 & K1 & NK1 & K1 & NK1 & K1 & NK1 \\
\hline $\mathrm{N}$ & 55 & 10 & 55 & 10 & 55 & 10 & 43 & 10 \\
\hline $\mathrm{N}(\mathrm{NA})$ & 1 & 1 & 1 & 1 & 1 & 1 & 13 & 1 \\
\hline Mean & 1.195 & 0.753 & 1.589 & 1.380 & 0.398 & 0.264 & 1.473 & 0.710 \\
\hline \multirow[t]{2}{*}{$\mathrm{SD}$} & 0.940 & 0.296 & 1.618 & 1.538 & 0.373 & 0.223 & 2.009 & 0.616 \\
\hline & \multicolumn{2}{|c|}{ K1 - NK1 } & \multicolumn{2}{|c|}{ K1 $1-$ NK1 } & \multicolumn{2}{|c|}{ K1 - NK1 } & \multicolumn{2}{|c|}{ K1 - NK1 } \\
\hline Difference & \multicolumn{2}{|c|}{0.248} & \multicolumn{2}{|c|}{0.157} & \multicolumn{2}{|c|}{0.080} & \multicolumn{2}{|c|}{0.410} \\
\hline$P$-value & \multicolumn{2}{|c|}{0.094} & \multicolumn{2}{|c|}{0.537} & \multicolumn{2}{|c|}{0.321} & \multicolumn{2}{|c|}{0.055} \\
\hline $95 \%$ CI & -0.038 & 0.545 & -0.355 & 0.675 & -0.109 & 0.273 & -0.007 & 0.887 \\
\hline
\end{tabular}

Suitability of the DNA adducts level determination is shown in table 6 as well. Note: The group of non-smokers with diagnosis of COPD represents the fromer smokers (the total number of cigarettes smoked did not exceed 100,000 units).

Key to tables 1-6

$\mathrm{N} \quad$ Total number of subjects

N(NA) Number of missing subjects

Mean Arithmetic mean

SD Standard deviation

Difference Nonparametric estimate of difference between groups

$P$-value P-value for the difference between groups. Calculated using non-parametric Mann-Whitney-Wilcoxon test with normal approximation.

95\% CI Two tailed 95\% Confidence interval for the difference between groups

All values are in Arbitrary Units (A.U.)

Table 7. Assessment of possible association for IgA versus vitamin intake

\begin{tabular}{|c|c|c|c|c|c|c|c|c|c|}
\hline & Mean & SD & $\mathbf{0} \%$ & $25 \%$ & $50 \%$ & $75 \%$ & $100 \%$ & $\mathbf{N}$ & NA \\
\hline 0 & 1.610675 & 1.605979 & 0.18924303 & 0.7161572 & 1.116402 & 1.698413 & 8.407407 & 85 & 1 \\
\hline 1 & 1.677947 & 1.431106 & 0.01101591 & 0.9028078 & 1.323725 & 1.862278 & 13.039344 & 167 & 1 \\
\hline 2 & 1.445345 & 1.137493 & 0.17821782 & 0.8512613 & 1.138394 & 1.679445 & 9.133038 & 92 & 1 \\
\hline 3 & 1.170338 & 1.033218 & 0.13368984 & 0.5483871 & 0.797861 & 1.432425 & 5.370370 & 56 & 3 \\
\hline
\end{tabular}

Key to table 7:

Mean Arithmetic mean

SD Standard deviation

0\% Minimum

$25 \% \quad 1^{\text {st }}$ quartile

$50 \% \quad$ Median

$75 \% \quad 3^{\text {rd }}$ quartile

$100 \% \quad$ Maximum

$\mathrm{N} \quad$ Number of subjects

NA Number of missing subjects

Note: A certain tendency of IgA decrease with increasing vitamin consumption 
comparing patients with cancerous and non-cancerous lung disease (COPD) (see Table 4).

After redistribution of results based on individual groups, we have found increased levels of DNA adducts in smoking patients with COPD in comparison with non-smoking patients and those with cancerous lung disease (see Table 5 and 6).

Both lung cancer diagnoses and COPD, presents a very serious lung diseases that are unfortunately most often diagnosed in advanced stages, when changes in the levels of the tested parameters might no longer be visible. Moreover, it is necessary to take into account the fact that while COPD is a disease in the aetiology of which a long-term smoking and exposure of the respiratory tracts to toxic substances plays a crucial role, the aetiology of cancerous diseases is multi-factorial.

Furthermore, based on our clinical experience, we conclude that patients with COPD are almost always hospitalized due to occurring shortness of breath and manifestations of hypoxia while at the forefront of clinical problems in lung cancer is most commonly a manifestation of increased catabolism of the body (expressed in weakness, weight loss, fatigue and general cachexia), or failure of other organs caused by distant metastases. The immunological response of the long-term exhausted organism is with high probability altered.

\section{Conclusion}

Generally, we revealed no difference in antibody levels observed in patients in relation to cancerous or non-cancerous diseases.

Patients with diseases largely associated with smoking (COPD patients - smokers) showed increased levels of DNA adducts in comparison to non-smoking patients and those with cancerous lung disease.

There had also been confirmed the hypothesis that the genotoxic risk of $\mathrm{PAH}$ exposure increases with residence or otherwise lengthily staying in smoky or else PAHs polluted environments.

An important benefit of the epidemiological study is more accurate detection of effects of exposure to PAHs from the environment on the organism, which could allow initiating preventive measures already at the early stages of reversible changes in the organism.

In this study the relevance not only for the determination of each of the existing markers (DNA adducts), but also for determining new markers (anti-BPDE-DNA, anti-B(a)P IgA antibodies) that provide information on exposures to contaminating components of the environment and their effects on the organism was pointed out.
Acknowledgements: This work was supported by the Ministry of Education, Youth and Sports of the Czech Republic (Grant \# 2B06150).

\section{References}

[1] SUTA M Environmental chemical compoundd and health. ISBN 978-80-87308-00-4, online PDF (in czech)

[2] WOGAN GN, HECHT SS, FELTON JS, CONNEY AH, LOEB LA Environmental and chemical carcinogenesis. Semin Cancer Biol 2004; 14: 473-86. http://dx.doi.org/10.1016/ j.semcancer.2004.06.010

[3] RANDERATH E, MILLER RH, MITTAL D, AVITTS TA, DUNSFORD $\mathrm{H}$ A et al. DNA damage in tissues of cigaretce smokers as determined by 32P-postlabeling assay J Natl Cancer Indy 1989; 81: 341-347

[4] ROBBINS CS, DAWE DE, GONCHAROVA SI, POULADI MA, DRANNIK AG et al. Cigarette smoke decreases pulmonary dendritic cells and impacts antiviral immune responsiveness. Am J Respir Cell Mol Biol 2004;30: 202-211 http://dx.doi.org/10.1165/rcmb.2003-0259OC

[5] SAVAGE SM, DONALDSON LA, CHERIAN S., CHILUKURI $\mathrm{R}$, WHITE VA et al. Effects of cigarette smoke on the immune response. II. Chronic exposure to cigarette smoke inhibits surface immunoglobulin-mediated responses in B cells Toxicol Appl Pharmacol 1991;111: 523-529 http://dx.doi. org/10.1016/0041-008X(91)90256-E

[6] SATO H, AOKI Y Mutagenesis by environmental pollutants and bio-monitoring of environmental mutagens Curr Drug Metab 2002; 3: 311-319 http://dx.doi.org/10.2174/ $\underline{1389200023337603}$

[7] FARMER PB, SINGH R, KAUR B, SRAM RJ, BINKOVA B et al. Molecular epidemiology studies of carcinogenic environmental pollutants. Effects of polycyclic aromatic hydrocarbons (PAHs) in environmental pollution on exogenous and oxidative DNA damage Mutat Res 2003; 544: 397-402

[8] HAUGEN A, BECHER G, BENESTAD C, VAHAKANGAS $\mathrm{K}$, TRIVERS GE et al. Determination of polycyclic aromatic hydrocarbons in the urine, benzo(a)pyrene diol epoxide-DNA adducts in lymphocyte DNA, and antibodies to the adducts in sera from coke oven workers exposed to measured amounts of polycyclic aromatic hydrocarbons in the work atmosphere Cancer Res, 1986; 46: 4178-4183

[9] DE BUCK SS, BOUCHE FB, BRANDENBURGER A, MULLER CP Modulation of the metabolism and adverse effects of benzo[a]pyrene by a specific antibody: a novel host factor in environmental carcinogenesis? Carcinogenesis 2005; 26: 835-844 http://dx.doi.org/10.1093/carcin/bgi010

[10] CERNOHORSKA H, KLIMESOVA S, LEPSA L, JINOCH P, MILCOVA A et al. Influence of immunization with non-genotoxic PAH-KLH conjugates on the resistance of organisms exposed to benzo(a)pyrene Mut Res 2012; 742: 2-10 